\title{
On the backward Euler method for a generalized Ait-Sahalia-type rate model with Poisson jumps
}

\author{
Yuying Zhao · Xiaojie Wang · Mengchao Wang 凶
}

\begin{abstract}
This article aims to reveal the mean-square convergence rate of the backward Euler method (BEM) for a generalized Ait-Sahalia interest rate model with Poisson jumps. The main difficulty in the analysis is caused by the non-globally Lipschitz drift and diffusion coefficients of the model. We show that the BEM preserves the positivity of the original problem. Furthermore, we successfully recover the mean-square convergence rate of order one-half for the BEM. The theoretical findings are accompanied by several numerical examples.
\end{abstract}

Keywords Ait-Sahalia model, Poisson jumps, backward Euler method, mean-square convergence rate AMS subject classification: 60H35, 60H15, 65C 30 .

\section{Introduction}

As is well known, stochastic differential equations (SDEs) are widely used in various scientific areas to model real-life phenomena affected by random noises. However, in order to model the event-driven phenomena, it is necessary and significant to introduce SDEs with Poisson jumps [6, 24]. For instance, the stock price movements might suffer from sudden and significant impacts caused by unpredictable important events such as market crashes, announcements made by central banks, changes in credit rating, etc. Over the last decade, SDEs with jumps have become increasingly popular for modelling market fluctuations, both for risk management and option pricing purposes (see, e.g., [6]). Since the analytic solutions of nonlinear SDEs with jumps are rarely available, numerical approximations become a powerful tool to understand the behavior of the underlying problems. Motivated by this, a great deal of research papers are devoted to the interesting topic (see, e.g., 2, 5, 7, 9, 11, 13, 16, 19, 24, 25, 27, 29, ).

The present article is concerned with the mean-square convergence analysis of a time-stepping scheme for a generalized Ait-Sahalia interest rate model with Poisson jumps, which takes the form as follows,

$$
\mathrm{d} X_{t}=\left(a_{-1} X_{t}^{-1}-a_{0}+a_{1} X_{t}-a_{2} X_{t}^{\gamma}\right) \mathrm{d} t+b X_{t}^{\theta} \mathrm{d} W_{t}+\varphi\left(X_{t^{-}}\right) \mathrm{d} N_{t}, \quad X_{0}=x_{0}, \quad t>0 .
$$

Here, $a_{-1}, a_{0}, a_{1}, a_{2}, b>0, \theta, \gamma>1, \varphi: \mathbb{R} \rightarrow \mathbb{R}$ and $X_{t^{-}}:=\lim _{s \rightarrow t^{-}} X_{s}$. Moreover, we let $\left\{W_{t}\right\}_{t \in[0, \infty)}$ and $\left\{N_{t}\right\}_{t \in[0, \infty)}$ be a one-dimensional Brownian motion and a Poisson process with the jump intensity

Yuying Zhao

School of Mathematics and Statistics, Central South University, Changsha, China

E-mail: y.y.zhao68@csu.edu.cn

Xiaojie Wang

School of Mathematics and Statistics, Central South University, Changsha, China

E-mail: x.j.wang7@csu.edu.cn

Mengchao Wang $\square$

School of Mathematics and Statistics, Central South University, Changsha, China

E-mail: wangmengchao@csu.edu.cn

This work was supported by Natural Science Foundation of China (11671405, 11971488, 91630312), Innovation Program of Central South University(No.2019zzts397) and Natural Science Foundation of Hunan Province for Distinguished Young Scholars (2020JJ2040). 
$\lambda>0$, respectively, on a filtered probability space $\left(\Omega, \mathscr{F}, \mathbb{P},\{\mathscr{F} t\}_{t \geq 0}\right)$ with respect to the normal filtration $\left\{\mathscr{F}_{t}\right\}_{t \geq 0}$. The Brownian motion and the Poisson process are assumed to be independent with each other. By $\tilde{N}_{t}:=N_{t}-\lambda t, t \in[0, \infty)$ we denote the compensated Poisson process, which is also a martingale with respect to the normal filtration $\left\{\mathscr{F}_{t}\right\}_{t \geq 0}$. Recall that the generalized Ait-Sahalia interest rate model without jumps, i.e., $\varphi \equiv 0$, has been already numerically studied by [26], where strong convergence of the backward Euler method was proved, but without revealing any convergence rate. Taking the jump function $\varphi$ to be a linear one, Deng et.al., 8, examined the analytical properties of the model, including the positivity, boundedness and pathwise asymptotic estimates. Also, they applied the Euler-Maruyama (EM) method to the particular model and proved that the explicit scheme converges in probability to the true solution of the model. To the best of our knowledge, no strong convergence rate has been reported in the literature for numerical approximations of the generalized Ait-Sahalia interest rate model with Poisson jumps as 1 .

In this paper, we focus on the the backward Euler method for (1), which has been widely studied for SDEs without jumps (see [1, 14, 15, 21, 23, 28, 30]). When used to solve the aforementioned Ait-Sahalia model with jumps, the chosen scheme is shown to be positivity preserving. Furthermore we provide an easy approach of error analysis to successfully obtain its mean-square convergence rate of order one-half for full parameters in the case $\gamma+1>2 \theta$ and for parameters obeying $\frac{a_{2}}{b^{2}}>2 \gamma-\frac{3}{2}$ in the general critical case $\gamma+1=2 \theta$. As a byproduct, this work reveals the expected convergence rate for the model without jumps, which is missing in [26].

The remainder of this paper is organized as follows. The next section concerns the properties of the solution to the model, including the existence and uniqueness, the positivity and the moment boundedness of the solution. The mean-square convergence rate of the BEM is identified in section 3 for the generalized Ait-Sahalia interest rate model with Poisson jumps. Finally numerical experiments are performed to illustrate the theoretical results.

\section{The jump-extended Ait-Sahalia model}

Throughout this paper we will use the following notation. Let $|\cdot|$ and $\langle\cdot, \cdot\rangle$ be the Euclidean norm and the inner product in $\mathbb{R}$, respectively. Given a filtered probability space $\left(\Omega, \mathcal{F},\left\{\mathcal{F}_{t}\right\}_{t \in[0, T]}, \mathbb{P}\right)$ satisfying the usual hypotheses, that is to say, it is right continuous and increasing while $\mathcal{F}_{0}$ contains all $\mathbb{P}$-null sets. Let $\left\{W_{t}\right\}_{t \in[0, \infty)}$ and $\left\{N_{t}\right\}_{t \in[0, \infty)}$ be a one-dimensional Brownian motion and a scalar Poisson process with the jump intensity $\lambda>0$, respectively, with respect to the normal filtration $\left\{\mathscr{F}_{t}\right\}_{t \geq 0}$. Let $\mathbb{E}$ denote the expectation and let $L^{p}(\Omega ; \mathbb{R})$ denote the space consisting of $\mathbb{R}$-value $p$-times integrable random variables with the norm defined by $\|\xi\|_{L^{p}(\Omega ; \mathbb{R})}:=\left(\mathbb{E}\left[|\xi|^{p}\right]\right)^{1 / p}$ for any $p \geq 1$. Let $x \vee y:=\max \{x, y\}$ and $x \wedge y:=\min \{x, y\}$ for any $x, y \in \mathbb{R}$. For notational simplicity, the letter $C$ is used to denote a generic positive constant, which is independent of the time stepsize and may vary for each appearance. Additionally, we suppose that for the coefficient function $\varphi: \mathbb{R} \rightarrow \mathbb{R}$ in (1), there are constants $M>0$ and $\varepsilon_{0}>0$, such that

$$
|\varphi(x)-\varphi(y)| \leq M|x-y|, \quad \forall x, y>0
$$

and

$$
x+\varphi(x)>\varepsilon_{0} \min \{1, x\}, \quad \forall x>0 .
$$

We comment that the inequality (3) naturally holds when $x+\varphi(x)>\varepsilon_{0} x$ or $x+\varphi(x)>\varepsilon_{0}$ for some $\varepsilon_{0}>0$. Also, the inequality 2 immediately implies

$$
|\varphi(x)| \leq C(1+|x|), \quad \forall x>0
$$

where $C$ is a generic positive constant as explained above.

In the above setting, we attempt to show the existence and uniqueness of a positive global solution to the problem (1). The well-posedness of the Ait-Salalia model without jumps, i.e., $\varphi \equiv 0$, has been already asserted by [26, Theorem 2.1]. When the jump coefficient $\varphi$ is a linear function, the well-posedness of the solution to the model (1) has been obtained in [8, Theorem 2.3]. In what follows, we extend the analysis in [8, 26] to cope with a more general nonlinear jump coefficient. 
Proposition 1 Let conditions (2), (3) hold and let the initial data $X_{0}=x_{0}>0$. For constants $a_{-1}, a_{0}, a_{1}, a_{2}, b>0$ and $\gamma, \theta>1$, the problem (1) admits a unique positive global solution, which almost surely satisfies

$$
X_{t}=X_{0}+\int_{0}^{t}\left(a_{-1} X_{s}^{-1}-a_{0}+a_{1} X_{s}-a_{2} X_{s}^{\gamma}\right) d s+\int_{0}^{t} b X_{s}^{\theta} d W_{s}+\int_{0}^{t} \varphi\left(X_{s^{-}}\right) d N_{s}, \quad t \geq 0 .
$$

Proof of Proposition 1. It is easy to see that the drift and diffusion coefficients of (5) are locally Lipschitz continuous in $(0, \infty)$ and that the jump coefficient is globally Lipschitz continuous in $\mathbb{R}$. Following the standard truncation arguments [20] and noting $X_{0}=x_{0}>0$, one can show that there is a unique maximal local solution $X_{t}, t \in\left[0, \tau_{e}\right)$, where $\tau_{e}$ is the stopping time of the explosion or first zero time. To confirm the global solution, we need to prove $\tau_{e}=\infty$ a.s. For any sufficiently large positive integer $n$, satisfying $1 / n<x_{0}<n$, we define the stopping times

$$
\tau_{n}:=\inf \left\{t \in\left[0, \tau_{e}\right): X_{t} \notin(1 / n, n)\right\}
$$

where throughout this paper we set $\inf (\emptyset)=\infty$. Obviously $\tau_{n}$ is increasing as $n \rightarrow \infty$ and we set $\tau_{\infty}:=\lim _{n \rightarrow \infty} \tau_{n}$. In view of (6), one knows $\tau_{\infty} \leq \tau_{e}$ a.s. If we can prove $\tau_{n} \rightarrow \infty$ a.s. as $n \rightarrow \infty$, then $\tau_{e}=\infty$ a.s. and $x(t)>0$ a.s. for all $t \geq 0$, which completes the proof. To prove $\tau_{\infty}=\infty$ a.s., it suffices to show that $\mathbb{P}\left\{\tau_{n} \leq T\right\} \rightarrow 0$ as $n \rightarrow \infty$ for any constant $T>0$, which immediately implies $\mathbb{P}\left\{\tau_{\infty}=\infty\right\}=1$, as required.

Given a fixed constant $\alpha \in(0,1)$, let us define a function $V \in C^{2}((0, \infty),(0, \infty))$ by

$$
V(x)=x^{\alpha}-\alpha \log x .
$$

It is easy to check that $V(x) \rightarrow \infty$ as $x \rightarrow \infty$ or $x \rightarrow 0$ and that

$$
V_{x}(x)=\alpha\left(x^{\alpha-1}-x^{-1}\right), \quad V_{x x}(x)=\alpha(\alpha-1) x^{\alpha-2}+\alpha x^{-2} .
$$

For brevity we write

$$
\mu(x):=a_{-1} x^{-1}-a_{0}+a_{1} x-a_{2} x^{\gamma}, \quad \phi(x):=b x^{\theta},
$$

and introduce the diffusion operator $\mathbb{L} V:(0, \infty) \rightarrow \mathbb{R}$ defined by

$$
\mathbb{L} V(x)=V_{x}(x) \mu(x)+\frac{1}{2} V_{x x}(x) \phi^{2}(x) .
$$

Bearing (8) and (9) in mind, one can compute that

$$
\begin{aligned}
\mathbb{L} V(x)= & \alpha\left(x^{\alpha-1}-x^{-1}\right)\left(a_{-1} x^{-1}-a_{0}+a_{1} x-a_{2} x^{\gamma}\right)+\frac{b^{2}}{2}\left[\alpha(\alpha-1) x^{\alpha-2}+\alpha x^{-2}\right] x^{2 \theta} \\
= & a_{-1} \alpha x^{\alpha-2}-a_{0} \alpha x^{\alpha-1}+a_{1} \alpha x^{\alpha}-a_{2} \alpha x^{\alpha+\gamma-1}-a_{-1} \alpha x^{-2}+a_{0} \alpha x^{-1} \\
& -a_{1} \alpha+a_{2} \alpha x^{\gamma-1}-\frac{b^{2} \alpha(1-\alpha)}{2} x^{\alpha+2 \theta-2}+\frac{b^{2} \alpha}{2} x^{2 \theta-2} .
\end{aligned}
$$

Using (3) and (4), one can find a constant $c_{\lambda}$ depending on $\lambda$ such that

$$
\begin{aligned}
\lambda(V(x+\varphi(x))-V(x)) & =\lambda\left[(x+\varphi(x))^{\alpha}-\alpha \log (x+\varphi(x))-\left(x^{\alpha}-\alpha \log x\right)\right] \\
& =\lambda\left[(x+\varphi(x))^{\alpha}-x^{\alpha}-\alpha \log \left(\frac{x+\varphi(x)}{x}\right)\right] \\
& \leq c_{\lambda}\left(1+x^{\alpha}\right), \quad \forall x>0 .
\end{aligned}
$$

Taking (11) and (12) into account and recalling $0<\alpha<1, \gamma>1, \theta>1, \lambda>0$, one can find a constant $K_{1}>0$ such that

$$
\sup _{x \in(0, \infty)}(\mathbb{L} V(x)+\lambda(V(x+\varphi(x))-V(x))) \leq K_{1}<\infty .
$$

By the Itô formula [10] applied to $V\left(x\left(t \wedge \tau_{n}\right)\right), t \in[0, T]$, we infer

$$
\mathbb{E}\left[V\left(x\left(T \wedge \tau_{n}\right)\right)\right] \leq V\left(x_{0}\right)+K_{1} T<\infty, \quad \forall T>0
$$


Owing to the definitions (6), (7), we deduce from the above estimate that

$$
\mathbb{P}\left(\tau_{n} \leq T\right)[V(1 / n) \wedge V(n)] \leq \mathbb{E} V\left(x\left(T \wedge \tau_{n}\right)\right) \leq V\left(x_{0}\right)+K_{1} T<\infty .
$$

This implies that $\lim _{n \rightarrow \infty} \mathbb{P}\left(\tau_{n} \leq T\right)=0$ for any constant $T>0$ and the proof is thus complete.

In the following error analysis, the moment bounds of the analytic solution are frequently used. The next lemma indicates when $p$-th moments of the solution to (1) are bounded.

Lemma 1 Let all conditions in Proposition 1 hold and let $\left\{X_{t}\right\}_{t \geq 0}$ be the unique solution to (1), given by (5). If one of the following two conditions holds:

(i) $p \geq 2$ when $\gamma+1>2 \theta$;

(ii) $2 \leq p<\frac{2 a_{2}+b^{2}}{b^{2}}$ when $\gamma+1=2 \theta$, then

$$
\sup _{t \in[0, \infty)} \mathbb{E}\left[\left|X_{t}\right|^{p}\right]<\infty
$$

Proof of Lemma 1. For a sufficiently large positive integer $n$ satisfying $\frac{1}{n}<x_{0}<n$, we define the stopping time

$$
\tau_{n}:=\inf \left\{t \in[0, \infty): X_{t} \notin(1 / n, n)\right\} .
$$

Also, we define $V_{1}: \mathbb{R}_{+} \times[0, \infty) \rightarrow \mathbb{R}_{+}$as follows

$$
V_{1}(x, t):=e^{t} x^{p}, \quad x \in \mathbb{R}_{+}, t \in[0, \infty) .
$$

We compute that

$$
\begin{aligned}
& \mathbb{L} V_{1}(x, t)+\lambda\left(V_{1}(x+\varphi(x), t)-V_{1}(x, t)\right) \\
= & e^{t}\left[p x^{p-1}\left(a_{-1} x^{-1}-a_{0}+a_{1} x-a_{2} x^{\gamma}\right)+\frac{1}{2} b^{2} p(p-1) x^{p-2+2 \theta}\right] \\
& +\lambda e^{t}\left((x+\varphi(x))^{p}-x^{p}\right) \\
= & e^{t}\left[a_{-1} p x^{p-2}-a_{0} p x^{p-1}+a_{1} p x^{p}-a_{2} p x^{p+\gamma-1}+\frac{1}{2} b^{2} p(p-1) x^{p-2+2 \theta}\right. \\
& \left.+\lambda\left((x+\varphi(x))^{p}-x^{p}\right)\right],
\end{aligned}
$$

where the diffusion operator $\mathbb{L} V_{1}: \mathbb{R}_{+} \times[0, \infty) \rightarrow \mathbb{R}_{+}$is defined by

$$
\mathbb{L} V_{1}(x, t):=\frac{\partial V_{1}(x, t)}{\partial x} \mu(x)+\frac{1}{2} \frac{\partial^{2} V_{1}(x, t)}{\partial x^{2}} \phi^{2}(x) .
$$

In light of condition (i) or (ii) as well as (3), (4), one can find a constant $K_{2}>0$, such that

$$
\mathbb{L} V_{1}\left(X_{t}\right)+\lambda\left(V_{1}\left(X_{t}+\varphi\left(X_{t}\right)\right)-V_{1}\left(X_{t}\right)\right) \leq K_{2} e^{t}
$$

Indeed, in the case (i), we can directly get $p+\gamma-1>p-2+2 \theta$. Thus, it is easy to see that the highest power of $x$ is $p+\gamma-1$ in (19). As a result of $a_{2}>0$, there is a constant $K_{2}>0$ such that 20 is fulfilled. In the case (ii), one can derive that $p+\gamma-1=p-2+2 \theta$. Furthermore, the inequality $\frac{1}{2} b^{2} p(p-1)-a_{2} p<0$ holds under the condition $p<\frac{2 a_{2}+b^{2}}{b^{2}}$. Similar to the above analysis, we can arrive at the same conclusion. By the Itô formula [10], for any $t \geq 0$,

$$
\mathbb{E}\left[e^{t \wedge \tau_{n}} X_{t \wedge \tau_{n}}^{p}\right] \leq x_{0}^{p}+K_{2} e^{t} .
$$

Letting $n \rightarrow \infty$ and applying Fatou's lemma, we obtain

$$
\mathbb{E}\left[\left|X_{t}\right|^{p}\right] \leq \frac{x_{0}^{p}}{e^{t}}+K_{2}, \quad t \geq 0 .
$$

The proof of Lemma 1 is thus completed.

The next lemma gives the inverse moment bounds of the solution to (1), which is also required in the error analysis.

Lemma 2 Let all conditions in Proposition 1 hold with $\gamma+1 \geq 2 \theta$ and let $\left\{X_{t}\right\}_{t \geq 0}$ be the unique solution to (1), given by (5). Then for any $p \geq \max \{1, \gamma-1\}$ it holds

$$
\sup _{t \in[0, \infty)} \mathbb{E}\left[\left|X_{t}\right|^{-p}\right]<\infty
$$


Proof of Lemma 2. Define $V_{2}: \mathbb{R}_{+} \times[0, \infty) \rightarrow \mathbb{R}_{+}$as follows

$$
V_{2}(x, t):=e^{t} x^{-p}, \quad x \in \mathbb{R}_{+}, \quad t \in[0, \infty) .
$$

Here, $\tau_{n}$ and the functional $\mathbb{L}$ have been defined in the proof of Lemma 1 . Then we have

$$
\begin{aligned}
& \mathbb{L} V_{2}(x, t)+\lambda\left(V_{2}(x+\varphi(x), t)-V_{2}(x, t)\right) \\
= & e^{t}\left[-a_{-1} p x^{-p-2}+a_{0} p x^{-p-1}-a_{1} p x^{-p}+a_{2} p x^{-p+\gamma-1}+\frac{1}{2} b^{2} p(p+1) x^{-p-2+2 \theta}\right. \\
& \left.+\lambda\left((x+\varphi(x))^{-p}-x^{-p}\right)\right] .
\end{aligned}
$$

Taking $\gamma+1 \geq 2 \theta$ and $\gamma \leq p+1$ into account promises that the highest power of $x$ on the right-hand side of 25) is $-p+\gamma-1 \leq 0$. Also, it is not difficult to check that $-p-2$ is the lowest power of $x$ in (25). Due to the negative coefficient of $x^{-p-2}$, i.e, $-a_{-1} p<0$, there exists a constant $K_{3}>0$ such that

$$
\mathbb{L} V_{2}\left(X_{t}\right)+\lambda\left(V_{2}\left(X_{t}+\varphi\left(X_{t}\right)\right)-V_{2}\left(X_{t}\right)\right) \leq K_{3} e^{t}
$$

The remaining proof is similar to that of Lemma 1 and thus omitted.

\section{Mean-square convergence rate of the backward Euler method for the generalized Ait-Sahalia-type rate model with Poisson jumps}

In this section we aim to propose and analyze the backward Euler method (BEM) for strong approximations of the Ait-Sahalia model with Poisson jumps (1). Given $T \in(0, \infty)$ and $N \in \mathbb{N}$, we construct a uniform mesh on the interval $[0, T]$ with the uniform stepsize $h=\frac{T}{N}$. Based on the uniform mesh, we propose a numerical scheme for (1) as follows:

$$
Y_{n}=Y_{n-1}+h\left[a_{-1} Y_{n}^{-1}-a_{0}+a_{1} Y_{n}-a_{2} Y_{n}^{\gamma}\right]+b Y_{n-1}^{\theta} \Delta W_{n-1}+\varphi\left(Y_{n-1}\right) \Delta N_{n-1}, \quad Y_{0}=X_{0},
$$

where $\Delta W_{n-1}:=W_{t_{n}}-W_{t_{n-1}}, \Delta N_{n-1}:=N_{t_{n}}-N_{t_{n-1}}, n \in\{1,2, \ldots, N\}, N \in \mathbb{N}$. Next we shall check that the numerical approximations produced by (27) are well defined and preserves positivity of the original model (1).

Lemma 3 Let all conditions in Proposition 1 hold. For any $h \leq \frac{1}{a_{1}}$, the BEM (27) is well-defined in the sense that it admits a unique positive solution.

Proof of Lemma 3. The proof is similar to that of [26, Lemma 3.1].

To carry out the error analysis of the BEM for the model, we treat the standard case $\kappa+1>2 \rho$ and the critical case $\kappa+1=2 \rho$ separately.

3.1 The case $\gamma+1>2 \theta$

This subsection attempts to recover the expected convergence rate of the BEM for the Ait-Sahalia model in the case $\gamma+1>2 \theta$. Before proceeding further, we present an important lemma, which plays an essential role in deriving the mean-square convergence rate of BEM.

Lemma 4 Let all conditions in Proposition 1 hold with $\gamma+1>2 \theta$ and let $\left\{X_{t}\right\}_{t \geq 0}$ be the process defined by (5). Then it holds that

$$
\left\|X_{t}-X_{s}\right\|_{L^{2}(\Omega ; \mathbb{R})} \leq C|t-s|^{\frac{1}{2}}, \quad 0 \leq t, s \leq T .
$$

Proof of Lemma 4. Recall that the coefficients of (1) are defined as follows:

$$
\mu(x):=a_{-1} x^{-1}-a_{0}+a_{1} x-a_{2} x^{\gamma}, \quad \phi(x):=b x^{\theta} .
$$

Letting $t>s$ without loss of generality, then one can easily check that

$$
\mathbb{E}\left[\left|X_{t}-X_{s}\right|^{2}\right]=\mathbb{E}\left[\left|\int_{s}^{t} \mu\left(X_{r}\right) \mathrm{d} r+\int_{s}^{t} \phi\left(X_{r}\right) \mathrm{d} W_{r}+\int_{s}^{t} \varphi\left(X_{r^{-}}\right) \mathrm{d} \tilde{N}_{r}+\lambda \int_{s}^{t} \varphi\left(X_{r^{-}}\right) \mathrm{d} r\right|^{2}\right] .
$$


Employing the Young inequality, the Hölder inequality, the Itô isometry and Lemmas 1, 2, one can arrive at

$$
\begin{aligned}
\mathbb{E}\left[\left|X_{t}-X_{s}\right|^{2}\right] \leq & 4|t-s| \int_{s}^{t} \mathbb{E}\left[\left|\mu\left(X_{r}\right)\right|^{2}\right] \mathrm{d} r+4 \int_{s}^{t} \mathbb{E}\left[\left|\phi\left(X_{r}\right)\right|^{2}\right] \mathrm{d} r \\
& +4 \lambda \int_{s}^{t} \mathbb{E}\left[\left|\varphi\left(X_{r^{-}}\right)\right|^{2}\right] \mathrm{d} r+4 \lambda^{2}|t-s| \int_{s}^{t} \mathbb{E}\left[\left|\varphi\left(X_{r^{-}}\right)\right|^{2}\right] \mathrm{d} r \\
\leq & C|t-s|^{2}+C|t-s| \\
\leq & C|t-s|
\end{aligned}
$$

as required.

Equipped with the above lemmas, we are ready to derive the mean-square convergence rate of order one-half for the scheme.

Theorem 1 Let all conditions in Proposition 1 hold with $\gamma+1>2 \theta$. Let $\left\{X_{t}\right\}_{0 \leq t \leq T}$ and $\left\{Y_{n}\right\}_{0 \leq n \leq N}$ be solutions to (1) and (27), respectively. Then for any $h \in\left(0, \frac{1}{2 L}\right)$ it holds that

$$
\sup _{N \in \mathbb{N}} \sup _{0 \leq n \leq N}\left\|Y_{n}-X_{t_{n}}\right\|_{L^{2}(\Omega ; \mathbb{R})} \leq C h^{\frac{1}{2}}
$$

where $L:=a_{1}+\frac{(q-1) b^{2} \theta^{2}(\gamma+1-2 \theta)}{2(\gamma-1)}\left(\frac{(q-1) b^{2} \theta^{2}(\theta-1)}{a_{2} \gamma(\gamma-1)}\right)^{\frac{2 \theta-2}{\gamma+1-2 \theta}}$ for any $q>2$.

Proof of Theorem 1. By the definition (1), we learn that

$$
X_{t_{n}}=X_{t_{n-1}}+\int_{t_{n-1}}^{t_{n}}\left(a_{-1} X_{s}^{-1}-a_{0}+a_{1} X_{s}-a_{2} X_{s}^{\gamma}\right) \mathrm{d} s+\int_{t_{n-1}}^{t_{n}} b X_{s}^{\theta} \mathrm{d} W_{s}+\int_{t_{n-1}}^{t_{n}} \varphi\left(X_{s^{-}}\right) \mathrm{d} N_{s} .
$$

Subtracting (33) from 27) leads to

$$
E_{n}=E_{n-1}+h \Delta \mu_{n}+\Delta \phi_{n-1} \Delta W_{n-1}+\Delta \varphi_{n-1} \Delta N_{n-1}+\mathcal{M}_{t_{n}}
$$

where for short we denote

$$
\begin{aligned}
& E_{n}:=Y_{n}-X_{t_{n}}, \quad \Delta \mu_{n}:=\mu\left(Y_{n}\right)-\mu\left(X_{t_{n}}\right) \\
& \Delta \phi_{n-1}:=\phi\left(Y_{n-1}\right)-\phi\left(X_{t_{n-1}}\right), \quad \Delta \varphi_{n-1}:=\varphi\left(Y_{n-1}\right)-\varphi\left(X_{t_{n-1}}\right) \\
& \mathcal{M}_{t_{n}}:=\int_{t_{n-1}}^{t_{n}} \mu\left(X_{t_{n}}\right)-\mu\left(X_{s}\right) \mathrm{d} s+\int_{t_{n-1}}^{t_{n}} \phi\left(X_{t_{n-1}}\right)-\phi\left(X_{s}\right) \mathrm{d} W_{s}+\int_{t_{n-1}}^{t_{n}} \varphi\left(X_{t_{n-1}}\right)-\varphi\left(X_{s^{-}}\right) \mathrm{d} N_{s}
\end{aligned}
$$

Further, one can deduce from (34) that

$$
\left|E_{n}-h \Delta \mu_{n}\right|^{2}=\left|E_{n-1}+\Delta \phi_{n-1} \Delta W_{n-1}+\Delta \varphi_{n-1} \Delta N_{n-1}+\mathcal{M}_{t_{n}}\right|^{2} .
$$

By direct calculation, one can recast 36 as

$$
\begin{aligned}
& \left|E_{n}\right|^{2}+\left|h \Delta \mu_{n}\right|^{2} \\
= & \left|E_{n-1}\right|^{2}+\left|\Delta \phi_{n-1} \Delta W_{n-1}\right|^{2}+\left|\Delta \varphi_{n-1} \Delta N_{n-1}\right|^{2}+\left|\mathcal{M}_{t_{n}}\right|^{2}+2\left\langle E_{n-1}, \Delta \phi_{n-1} \Delta W_{n-1}\right\rangle \\
& +2\left\langle E_{n-1}, \Delta \varphi_{n-1} \Delta N_{n-1}\right\rangle+2\left\langle E_{n-1}, \mathcal{M}_{t_{n}}\right\rangle+2\left\langle\Delta \phi_{n-1} \Delta W_{n-1}, \Delta \varphi_{n-1} \Delta N_{n-1}\right\rangle \\
& +2\left\langle\Delta \phi_{n-1} \Delta W_{n-1}, \mathcal{M}_{t_{n}}\right\rangle+2\left\langle\Delta \varphi_{n-1} \Delta N_{n-1}, \mathcal{M}_{t_{n}}\right\rangle+2 h\left\langle E_{n}, \Delta \mu_{n}\right\rangle .
\end{aligned}
$$

Taking expectations on both sides of (37), one can easily arrive at

$$
\begin{aligned}
& \mathbb{E}\left[\left|E_{n}\right|^{2}\right]+h^{2} \mathbb{E}\left[\left|\Delta \mu_{n}\right|^{2}\right] \\
= & \mathbb{E}\left[\left|E_{n-1}\right|^{2}\right]+\mathbb{E}\left[\left|\Delta \phi_{n-1} \Delta W_{n-1}\right|^{2}\right]+\mathbb{E}\left[\left|\Delta \varphi_{n-1} \Delta N_{n-1}\right|^{2}\right]+\mathbb{E}\left[\left|\mathcal{M}_{t_{n}}\right|^{2}\right] \\
& +2 \mathbb{E}\left[\left\langle E_{n-1}, \Delta \phi_{n-1} \Delta W_{n-1}\right\rangle\right]+2 \mathbb{E}\left[\left\langle E_{n-1}, \Delta \varphi_{n-1} \Delta N_{n-1}\right\rangle\right] \\
& +2 \mathbb{E}\left[\left\langle E_{n-1}, \mathcal{M}_{t_{n}}\right\rangle\right]+2 \mathbb{E}\left[\left\langle\Delta \phi_{n-1} \Delta W_{n-1}, \Delta \varphi_{n-1} \Delta N_{n-1}\right\rangle\right] \\
& +2 \mathbb{E}\left[\left\langle\Delta \phi_{n-1} \Delta W_{n-1}, \mathcal{M}_{t_{n}}\right\rangle\right]+2 \mathbb{E}\left[\left\langle\Delta \varphi_{n-1} \Delta N_{n-1}, \mathcal{M}_{t_{n}}\right\rangle\right]+2 h \mathbb{E}\left[\left\langle E_{n}, \Delta \mu_{n}\right\rangle\right] .
\end{aligned}
$$


Before proceeding further, we claim that

$$
\mathbb{E}\left[\left|\mathcal{M}_{t_{n}}\right|^{2}\right]<\infty
$$

Indeed, using the Young inequality, the Hölder inequality, the Itô isometry and Lemmas 1 , 2 shows

$$
\begin{aligned}
\mathbb{E}\left[\left|\mathcal{M}_{t_{n}}\right|^{2}\right]= & \mathbb{E}\left[\mid \int_{t_{n-1}}^{t_{n}} \mu\left(X_{t_{n}}\right)-\mu\left(X_{s}\right) \mathrm{d} s+\int_{t_{n-1}}^{t_{n}} \phi\left(X_{t_{n-1}}\right)-\phi\left(X_{s}\right) \mathrm{d} W_{s}\right. \\
& \left.+\int_{t_{n-1}}^{t_{n}} \varphi\left(X_{t_{n-1}}\right)-\left.\varphi\left(X_{s^{-}}\right) \mathrm{d} N_{s}\right|^{2}\right] \\
\leq & 4 \mathbb{E}\left[\left|\int_{t_{n-1}}^{t_{n}} \mu\left(X_{t_{n}}\right)-\mu\left(X_{s}\right) \mathrm{d} s\right|^{2}\right]+4 \mathbb{E}\left[\left|\int_{t_{n-1}}^{t_{n}} \phi\left(X_{t_{n-1}}\right)-\phi\left(X_{s}\right) \mathrm{d} W_{s}\right|^{2}\right] \\
& +4 \mathbb{E}\left[\left|\int_{t_{n-1}}^{t_{n}} \varphi\left(X_{t_{n-1}}\right)-\varphi\left(X_{s^{-}}\right) \mathrm{d} \tilde{N}_{s}\right|^{2}\right]+4 \mathbb{E}\left[\lambda^{2}\left|\int_{t_{n-1}}^{t_{n}} \varphi\left(X_{t_{n-1}}\right)-\varphi\left(X_{s^{-}}\right) \mathrm{d} s\right|^{2}\right] \\
\leq & 4 h \int_{t_{n-1}}^{t_{n}} \mathbb{E}\left[\left|\mu\left(X_{t_{n}}\right)-\mu\left(X_{s}\right)\right|^{2}\right] \mathrm{d} s+4 \int_{t_{n-1}}^{t_{n}} \mathbb{E}\left[\left|\phi\left(X_{t_{n-1}}\right)-\phi\left(X_{s}\right)\right|^{2}\right] \mathrm{d} s \\
& +4 \lambda \int_{t_{n-1}}^{t_{n}} \mathbb{E}\left[\left|\varphi\left(X_{t_{n-1}}\right)-\varphi\left(X_{s^{-}}\right)\right|^{2}\right] \mathrm{d} s+4 \lambda^{2} h \int_{t_{n-1}}^{t_{n}} \mathbb{E}\left[\left|\varphi\left(X_{t_{n-1}}\right)-\varphi\left(X_{s^{-}}\right)\right|^{2}\right] \mathrm{d} s \\
< &
\end{aligned}
$$

In addition, we note that for any $\gamma+1>2 \theta$ and $q>2$,

$$
\sup _{x>0}\left(\mu^{\prime}(x)+\frac{q-1}{2}\left(\phi^{\prime}(x)\right)^{2}\right)=\sup _{x>0}\left(-a_{-1} x^{-2}+a_{1}-a_{2} \gamma x^{\gamma-1}+\frac{q-1}{2} b^{2} \theta^{2} x^{2 \theta-2}\right)<\infty,
$$

which in turn implies

$$
\langle x-y, \mu(x)-\mu(y)\rangle+\frac{q-1}{2}|\phi(x)-\phi(y)|^{2} \leq L|x-y|^{2}, \quad \forall x, y \in \mathbb{R}_{+},
$$

where $L:=a_{1}+\sup _{x \in(0, \infty)}\left(\frac{q-1}{2} b^{2} \theta^{2} x^{2 \theta-2}-\gamma a_{2} x^{\gamma-1}\right)=a_{1}+\frac{(q-1) b^{2} \theta^{2}(\gamma+1-2 \theta)}{2(\gamma-1)}\left(\frac{(q-1) b^{2} \theta^{2}(\theta-1)}{a_{2} \gamma(\gamma-1)}\right)^{\frac{2 \theta-2}{\gamma+1-2 \theta}}$. As a direct result of (42), one can infer from (38) that

$$
\begin{aligned}
& \quad(1-2 h L) \mathbb{E}\left[\left|E_{n}\right|^{2}\right]+h^{2} \mathbb{E}\left[\left|\Delta \mu_{n}\right|^{2}\right] \\
& \leq \mathbb{E}\left[\left|E_{n-1}\right|^{2}\right]+\mathbb{E}\left[\left|\Delta \phi_{n-1} \Delta W_{n-1}\right|^{2}\right]+\mathbb{E}\left[\left|\Delta \varphi_{n-1} \Delta N_{n-1}\right|^{2}\right]+\mathbb{E}\left[\left|\mathcal{M}_{t_{n}}\right|^{2}\right] \\
& \quad+2 \mathbb{E}\left[\left\langle E_{n-1}, \Delta \phi_{n-1} \Delta W_{n-1}\right\rangle\right]+2 \mathbb{E}\left[\left\langle E_{n-1}, \Delta \varphi_{n-1} \Delta N_{n-1}\right\rangle\right]+2 \mathbb{E}\left[\left\langle E_{n-1}, \mathcal{M}_{t_{n}}\right\rangle\right] \\
& \quad+2 \mathbb{E}\left[\left\langle\Delta \phi_{n-1} \Delta W_{n-1}, \Delta \varphi_{n-1} \Delta N_{n-1}\right\rangle\right] \\
& \quad+2 \mathbb{E}\left[\left\langle\Delta \phi_{n-1} \Delta W_{n-1}, \mathcal{M}_{t_{n}}\right\rangle\right]+2 \mathbb{E}\left[\left\langle\Delta \varphi_{n-1} \Delta N_{n-1}, \mathcal{M}_{t_{n}}\right\rangle\right] .
\end{aligned}
$$

In the next step, we declare that

$$
\mathbb{E}\left[\left|E_{k}\right|^{2}\right]<\infty, \quad \mathbb{E}\left[\left|\Delta \mu_{k}\right|^{2}\right]<\infty, \quad \forall k \in\{0,1,2, \ldots, N\}, N \in \mathbb{N},
$$

whose proof relies on the mathematical induction argument. In fact, for $k=0, Y_{0}=X_{0}$ and thus $E_{0}=\Delta \mu_{0}=0$, the assertion naturally holds. Next we assume, for some $n \leq N$,

$$
\mathbb{E}\left[\left|E_{n-1}\right|^{2}\right]<\infty, \quad \mathbb{E}\left[\left|\Delta \mu_{n-1}\right|^{2}\right]<\infty
$$

In view of 2 and 42 , one can show that

$$
\begin{aligned}
& \mathbb{E}\left[\left|\Delta \varphi_{n-1}\right|^{2}\right] \leq M^{2} \mathbb{E}\left[\left|E_{n-1}\right|^{2}\right]<\infty, \\
& \mathbb{E}\left[\left|\Delta \phi_{n-1}\right|^{2}\right] \leq \frac{1}{q-1}(2 L+1) \mathbb{E}\left[\left|E_{n-1}\right|^{2}\right]+\frac{1}{q-1} \mathbb{E}\left[\left|\Delta \mu_{n-1}\right|^{2}\right]<\infty .
\end{aligned}
$$

Employing these bounded moments above enables us to deduce that

$$
\begin{aligned}
& \mathbb{E}\left[\left|\Delta \phi_{n-1} \Delta W_{n-1}\right|^{2}\right]=h \mathbb{E}\left[\left|\Delta \phi_{n-1}\right|^{2}\right], \\
& \mathbb{E}\left[\left\langle E_{n-1}, \Delta \phi_{n-1} \Delta W_{n-1}\right\rangle\right]=0, \\
& \mathbb{E}\left[\left\langle\Delta \phi_{n-1} \Delta W_{n-1}, \Delta \varphi_{n-1} \Delta N_{n-1}\right\rangle\right]=0,
\end{aligned}
$$


and

$$
\begin{aligned}
& \mathbb{E}\left[\left|\Delta \varphi_{n-1} \Delta N_{n-1}\right|^{2}\right]=\mathbb{E}\left[\left|\Delta \varphi_{n-1}\right|^{2}\right] \cdot \mathbb{E}\left[\left|\Delta N_{n-1}\right|^{2}\right] \\
& =\mathbb{E}\left[\left|\Delta \varphi_{n-1}\right|^{2}\right] \cdot\left(\mathbb{E}\left[\left|\Delta \tilde{N}_{n-1}\right|^{2}\right]+\mathbb{E}\left[|\lambda h|^{2}\right]+2 \mathbb{E}\left[\lambda h \Delta \tilde{N}_{n-1}\right]\right) \\
& =\left(\lambda h+\lambda^{2} h^{2}\right) \mathbb{E}\left[\left|\Delta \varphi_{n-1}\right|^{2}\right], \\
& \begin{aligned}
\mathbb{E}\left[\left\langle E_{n-1}, \Delta \varphi_{n-1} \Delta N_{n-1}\right\rangle\right] & =\mathbb{E}\left[\left\langle E_{n-1}, \Delta \varphi_{n-1} \Delta \tilde{N}_{n-1}\right\rangle\right]+\lambda h \mathbb{E}\left[\left\langle E_{n-1}, \Delta \varphi_{n-1}\right\rangle\right] \\
& =\lambda h \mathbb{E}\left[\left\langle E_{n-1}, \Delta \varphi_{n-1}\right\rangle\right],
\end{aligned}
\end{aligned}
$$

where $\Delta \tilde{N}_{n-1}:=\tilde{N}_{t_{n}}-\tilde{N}_{t_{n-1}}$. Therefore, using 40, 43, 45, 46, and the Cauchy-Schwarz inequality, we have

$$
\begin{aligned}
& (1-2 h L) \mathbb{E}\left[\left|E_{n}\right|^{2}\right]+h^{2} \mathbb{E}\left[\left|\Delta \mu_{n}\right|^{2}\right] \\
\leq & \mathbb{E}\left[\left|E_{n-1}\right|^{2}\right]+h \mathbb{E}\left[\left|\Delta \phi_{n-1}\right|^{2}\right]+\left(\lambda h+\lambda^{2} h^{2}\right) \mathbb{E}\left[\left|\Delta \varphi_{n-1}\right|^{2}\right]+\mathbb{E}\left[\left|\mathcal{M}_{t_{n}}\right|^{2}\right] \\
& +2 \lambda h \mathbb{E}\left[\left\langle E_{n-1}, \Delta \varphi_{n-1}\right\rangle\right]+\mathbb{E}\left[\left|E_{n-1}\right|^{2}\right]+\mathbb{E}\left[\left|\mathcal{M}_{t_{n}}\right|^{2}\right]+h \mathbb{E}\left[\left|\Delta \phi_{n-1}\right|^{2}\right] \\
& +\mathbb{E}\left[\left|\mathcal{M}_{t_{n}}\right|^{2}\right]+\left(\lambda h+\lambda^{2} h^{2}\right) \mathbb{E}\left[\left|\Delta \varphi_{n-1}\right|^{2}\right]+\mathbb{E}\left[\left|\mathcal{M}_{t_{n}}\right|^{2}\right] \\
\leq & (2+\lambda h) \mathbb{E}\left[\left|E_{n-1}\right|^{2}\right]+2 h \mathbb{E}\left[\left|\Delta \phi_{n-1}\right|^{2}\right]+\left(3 \lambda h+2 \lambda^{2} h^{2}\right) \mathbb{E}\left[\left|\Delta \varphi_{n-1}\right|^{2}\right]+4 \mathbb{E}\left[\left|\mathcal{M}_{t_{n}}\right|^{2}\right]<\infty
\end{aligned}
$$

This implies

$$
\mathbb{E}\left[\left|E_{n}\right|^{2}\right]<\infty, \quad \mathbb{E}\left[\left|\Delta \mu_{n}\right|^{2}\right]<\infty
$$

and the claim (44) is thus validated based on the mathematical induction argument. Similar to (46), we use (44) to infer

$$
\mathbb{E}\left[\left|\Delta \varphi_{k}\right|^{2}\right]<\infty, \quad \mathbb{E}\left[\left|\Delta \phi_{k}\right|^{2}\right]<\infty, \quad \forall k \in\{0,1,2, \ldots, N\}, N \in \mathbb{N} .
$$

At the moment we are well-prepared to prove the desired error estimate (32). Using (2), (42), (47), 48), (49), the Cauchy-Schwarz inequality and the properties of the conditional expectation, we deduce from (38) that

$$
\begin{aligned}
& \mathbb{E}\left[\left|E_{n}\right|^{2}\right]-\mathbb{E}\left[\left|E_{n-1}\right|^{2}\right] \\
\leq & h \mathbb{E}\left[\left|\Delta \phi_{n-1}\right|^{2}\right]+\left(\lambda h+\lambda^{2} h^{2}\right) \mathbb{E}\left[\left|\Delta \varphi_{n-1}\right|^{2}\right]+\mathbb{E}\left[\left|\mathcal{M}_{t_{n}}\right|^{2}\right]+\lambda h \mathbb{E}\left[\left|E_{n-1}\right|^{2}\right] \\
& +\lambda h \mathbb{E}\left[\left|\Delta \varphi_{n-1}\right|^{2}\right]+2 \mathbb{E}\left[\left\langle E_{n-1}, \mathcal{M}_{t_{n}}\right\rangle\right]+(q-2) h \mathbb{E}\left[\left|\Delta \phi_{n-1}\right|^{2}\right]+\frac{1}{q-2} \mathbb{E}\left[\left|\mathcal{M}_{t_{n}}\right|^{2}\right] \\
& +\left(\lambda h+\lambda^{2} h^{2}\right) \mathbb{E}\left[\left|\Delta \varphi_{n-1}\right|^{2}\right]+\mathbb{E}\left[\left|\mathcal{M}_{t_{n}}\right|^{2}\right]+2 h \mathbb{E}\left[\left\langle E_{n}, \Delta \mu_{n}\right\rangle\right] \\
\leq & \lambda h \mathbb{E}\left[\left|E_{n-1}\right|^{2}\right]+(q-1) h \mathbb{E}\left[\left|\Delta \phi_{n-1}\right|^{2}\right]+\left(3 \lambda h+2 \lambda^{2} h^{2}\right) \mathbb{E}\left[\left|\Delta \varphi_{n-1}\right|^{2}\right] \\
& +2 \mathbb{E}\left[\left\langle E_{n-1}, \mathbb{E}\left(\mathcal{M}_{t_{n}} \mid \mathcal{F}_{t_{n-1}}\right)\right\rangle\right]+\frac{2 q-3}{q-2} \mathbb{E}\left[\left|\mathcal{M}_{t_{n}}\right|^{2}\right]+2 h \mathbb{E}\left[\left\langle E_{n}, \Delta \mu_{n}\right\rangle\right] \\
\leq & \left(\lambda h+\left(3 \lambda h+2 \lambda^{2} h^{2}\right) M^{2}\right) \mathbb{E}\left[\left|E_{n-1}\right|^{2}\right]+(q-1) h \mathbb{E}\left[\left|\Delta \phi_{n-1}\right|^{2}\right]+h \mathbb{E}\left[\left|E_{n-1}\right|^{2}\right] \\
& +h^{-1} \mathbb{E}\left[\left|\mathbb{E}\left(\mathcal{M}_{t_{n}} \mid \mathcal{F}_{t_{n-1}}\right)\right|^{2}\right]+\frac{2 q-3}{q-2} \mathbb{E}\left[\left|\mathcal{M}_{t_{n}}\right|^{2}\right]+2 L h \mathbb{E}\left[\left|E_{n}\right|^{2}\right]-(q-1) h \mathbb{E}\left[\left|\Delta \phi_{n}\right|^{2}\right] \\
= & \left((\lambda+1) h+\left(3 \lambda h+2 \lambda^{2} h^{2}\right) M^{2}\right) \mathbb{E}\left[\left|E_{n-1}\right|^{2}\right]+(q-1) h \mathbb{E}\left[\left|\Delta \phi_{n-1}\right|^{2}\right] \\
& +h^{-1} \mathbb{E}\left[\left|\mathbb{E}\left(\mathcal{M}_{t_{n}} \mid \mathcal{F}_{t_{n-1}}\right)\right|^{2}\right]+\frac{2 q-3}{q-2} \mathbb{E}\left[\left|\mathcal{M}_{t_{n}}\right|^{2}\right]+2 L h \mathbb{E}\left[\left|E_{n}\right|^{2}\right]-(q-1) h \mathbb{E}\left[\left|\Delta \phi_{n}\right|^{2}\right] .
\end{aligned}
$$

Summing both sides of the above inequality from 1 to $n$, we get

$$
\begin{aligned}
\mathbb{E}\left[\left|E_{n}\right|^{2}\right] \leq & 2 L h \mathbb{E}\left[\left|E_{n}\right|^{2}\right]+\left(2 L h+(\lambda+1) h+\left(3 \lambda h+2 \lambda^{2} h^{2}\right) M^{2}\right) \sum_{k=0}^{n-1} \mathbb{E}\left[\left|E_{k}\right|^{2}\right] \\
& +h^{-1} \sum_{k=1}^{n} \mathbb{E}\left[\left|\mathbb{E}\left(\mathcal{M}_{t_{k}} \mid \mathcal{F}_{t_{k-1}}\right)\right|^{2}\right]+\frac{2 q-3}{q-2} \sum_{k=1}^{n} \mathbb{E}\left[\left|\mathcal{M}_{t_{k}}\right|^{2}\right]-(q-1) h \mathbb{E}\left[\left|\Delta \phi_{n}\right|^{2}\right] \\
\leq & 2 L h \mathbb{E}\left[\left|E_{n}\right|^{2}\right]+C h \sum_{k=0}^{n-1} \mathbb{E}\left[\left|E_{k}\right|^{2}\right] \\
& +h^{-1} \sum_{k=1}^{n} \mathbb{E}\left[\left|\mathbb{E}\left(\mathcal{M}_{t_{k}} \mid \mathcal{F}_{t_{k-1}}\right)\right|^{2}\right]+\frac{2 q-3}{q-2} \sum_{k=1}^{n} \mathbb{E}\left[\left|\mathcal{M}_{t_{k}}\right|^{2}\right] .
\end{aligned}
$$


Noting $1-2 L h>\zeta>0$ for some $\zeta>0$ and invoking the Gronwall inequality gives

$$
\mathbb{E}\left[\left|E_{n}\right|^{2}\right] \leq C\left(h^{-1} \sum_{j=1}^{n} \mathbb{E}\left[\left|\mathbb{E}\left(\mathcal{M}_{t_{j}} \mid \mathcal{F}_{t_{j-1}}\right)\right|^{2}\right]+\sum_{j=1}^{n} \mathbb{E}\left[\left|\mathcal{M}_{t_{j}}\right|^{2}\right]\right)
$$

Accordingly, it remains to estimate $\mathbb{E}\left[\left|\mathcal{M}_{t_{j}}\right|^{2}\right]$ and $\mathbb{E}\left[\left|\mathbb{E}\left(\mathcal{M}_{t_{j}} \mid \mathcal{F}_{t_{j-1}}\right)\right|^{2}\right]$ before attaining the mean-square convergence rate. An elementary inequality gives

$$
\begin{aligned}
\left\|\mathcal{M}_{t_{j}}\right\|_{L^{2}(\Omega ; \mathbb{R})}= & \| \int_{t_{j-1}}^{t_{j}} \mu\left(X_{t_{j}}\right)-\mu\left(X_{s}\right) \mathrm{d} s+\int_{t_{j-1}}^{t_{j}} \phi\left(X_{t_{j-1}}\right)-\phi\left(X_{s}\right) \mathrm{d} W_{s} \\
& +\int_{t_{j-1}}^{t_{j}} \varphi\left(X_{t_{j-1}}\right)-\varphi\left(X_{s^{-}}\right) \mathrm{d} N_{s} \|_{L^{2}(\Omega ; \mathbb{R})} \\
\leq & C \int_{t_{j-1}}^{t_{j}}\left\|X_{t_{j}}^{-1}-X_{s}^{-1}\right\|_{L^{2}(\Omega ; \mathbb{R})}+\left\|X_{t_{j}}-X_{s}\right\|_{L^{2}(\Omega ; \mathbb{R})}+\left\|X_{t_{j}}^{\gamma}-X_{s}^{\gamma}\right\|_{L^{2}(\Omega ; \mathbb{R})} \mathrm{d} s \\
& +b\left\|\int_{t_{j-1}}^{t_{j}} X_{t_{j-1}}^{\theta}-X_{s}^{\theta} \mathrm{d} W_{s}\right\|_{L^{2}(\Omega ; \mathbb{R})}+\left\|\int_{t_{j-1}}^{t_{j}} \varphi\left(X_{t_{j-1}}\right)-\varphi\left(X_{s^{-}}\right) \mathrm{d} N_{s}\right\|_{L^{2}(\Omega ; \mathbb{R})} .
\end{aligned}
$$

As a result of Lemma 4, we know

$$
\left\|X_{t_{j}}-X_{s}\right\|_{L^{2}(\Omega ; \mathbb{R})} \leq C h^{\frac{1}{2}}
$$

In addition, using the generalized Itô formula [10], the Young inequality, the Hölder inequality, the Itô isometry and Lemmas 1, 2, we can obtain that

$$
\begin{aligned}
& \left\|X_{t_{j}}^{\gamma}-X_{s}^{\gamma}\right\|_{L^{2}(\Omega ; \mathbb{R})}^{2} \\
= & \| \int_{s}^{t_{j}} \gamma \mu\left(X_{r}\right) X_{r}^{\gamma-1} \mathrm{~d} r+\int_{s}^{t_{j}} \gamma \phi\left(X_{r}\right) X_{r}^{\gamma-1} \mathrm{~d} W_{r}+\frac{1}{2} \gamma(\gamma-1) \int_{s}^{t_{j}} X_{r}^{\gamma-2} \phi^{2}\left(X_{r}\right) \mathrm{d} r \\
& +\int_{s^{+}}^{t_{j}}\left[\left(X_{r^{-}}+\varphi\left(X_{r^{-}}\right)\right)^{\gamma}-X_{r^{-}}^{\gamma}\right] \mathrm{d} N_{r} \|_{L^{2}(\Omega ; \mathbb{R})}^{2} \\
\leq & 4 \gamma^{2}\left\|\int_{s}^{t_{j}} \mu\left(X_{r}\right) X_{r}^{\gamma-1} \mathrm{~d} r\right\|_{L^{2}(\Omega ; \mathbb{R})}^{2}+4 \gamma^{2}\left\|\int_{s}^{t_{j}} \phi\left(X_{r}\right) X_{r}^{\gamma-1} \mathrm{~d} W_{r}\right\|_{L^{2}(\Omega ; \mathbb{R})}^{2} \\
& +\gamma^{2}(\gamma-1)^{2}\left\|\int_{s}^{t_{j}} X_{r}^{\gamma-2} \phi^{2}\left(X_{r}\right) \mathrm{d} r\right\|_{L^{2}(\Omega ; \mathbb{R})}^{2}+4\left\|\int_{s^{+}}^{t_{j}}\left[\left(X_{r^{-}}+\varphi\left(X_{r^{-}}\right)\right)^{\gamma}-X_{r^{-}}^{\gamma}\right] \mathrm{d} N_{r}\right\|_{L^{2}(\Omega ; \mathbb{R})}^{2} \\
\leq & 4 \gamma^{2} h \int_{s}^{t_{j}} \mathbb{E}\left[\left|\mu\left(X_{r}\right) X_{r}^{\gamma-1}\right|^{2}\right] \mathrm{d} r+4 \gamma^{2} \int_{s}^{t_{j}} \mathbb{E}\left[\left|\phi\left(X_{r}\right) X_{r}^{\gamma-1}\right|^{2}\right] \mathrm{d} r \\
& +\gamma^{2}(\gamma-1)^{2} h \int_{s}^{t_{j}} \mathbb{E}\left[\left|X_{r}^{\gamma-2} \phi^{2}\left(X_{r}\right)\right|^{2}\right] \mathrm{d} r+8 \lambda \int_{s^{+}}^{t_{j}} \mathbb{E}\left[\left|\left(X_{r^{-}}+\varphi\left(X_{r^{-}}\right)\right)^{\gamma}-X_{r^{-}}^{\gamma}\right|^{2}\right] \mathrm{d} r \\
& +8 \lambda^{2} h \int_{s^{+}}^{t_{j}} \mathbb{E}\left[\left|\left(X_{r^{-}}+\varphi\left(X_{r^{-}}\right)\right)^{\gamma}-X_{r^{-}}^{\gamma}\right|^{2}\right] \mathrm{d} r
\end{aligned}
$$

$\leq C h$ 
Similar to the proof of $(58)$, we can also derive that

$$
\begin{aligned}
& \left\|X_{t_{j}}^{-1}-X_{s}^{-1}\right\|_{L^{2}(\Omega ; \mathbb{R})}^{2} \\
& \leq 4\left\|\int_{s}^{t_{j}} \mu\left(X_{r}\right) X_{r}^{-2} \mathrm{~d} r\right\|_{L^{2}(\Omega ; \mathbb{R})}^{2}+4\left\|\int_{s}^{t_{j}} \phi\left(X_{r}\right) X_{r}^{-2} \mathrm{~d} W_{r}\right\|_{L^{2}(\Omega ; \mathbb{R})}^{2} \\
& +4\left\|\int_{s}^{t_{j}} X_{r}^{-3} \phi^{2}\left(X_{r}\right) \mathrm{d} r\right\|_{L^{2}(\Omega ; \mathbb{R})}^{2}+4\left\|\int_{s^{+}}^{t_{j}}\left[\left(X_{r^{-}}+\varphi\left(X_{r^{-}}\right)\right)^{-1}-X_{r^{-}}^{-1}\right] \mathrm{d} N_{r}\right\|_{L^{2}(\Omega ; \mathbb{R})}^{2} \\
& \leq 4 h \int_{s}^{t_{j}} \mathbb{E}\left[\left|\mu\left(X_{r}\right) X_{r}^{-2}\right|^{2}\right] \mathrm{d} r+4 \int_{s}^{t_{j}} \mathbb{E}\left[\left|\phi\left(X_{r}\right) X_{r}^{-2}\right|^{2}\right] \mathrm{d} r+4 h \int_{s}^{t_{j}} \mathbb{E}\left[\left|X_{r}^{-3} \phi^{2}\left(X_{r}\right)\right|^{2}\right] \mathrm{d} r \\
& +8 \lambda \int_{s^{+}}^{t_{j}} \mathbb{E}\left[\left|\frac{\varphi\left(X_{r^{-}}\right)}{\left(X_{r^{-}}+\varphi\left(X_{r^{-}}\right)\right) X_{r^{-}}}\right|^{2}\right] \mathrm{d} r+8 \lambda^{2} h \int_{s^{+}}^{t_{j}} \mathbb{E}\left[\left|\frac{\varphi\left(X_{r^{-}}\right)}{\left(X_{r^{-}}+\varphi\left(X_{r^{-}}\right)\right) X_{r^{-}}}\right|^{2}\right] \mathrm{d} r \\
& \leq 4 h \int_{s}^{t_{j}} \mathbb{E}\left[\left|\mu\left(X_{r}\right) X_{r}^{-2}\right|^{2}\right] \mathrm{d} r+4 \int_{s}^{t_{j}} \mathbb{E}\left[\left|\phi\left(X_{r}\right) X_{r}^{-2}\right|^{2}\right] \mathrm{d} r+4 h \int_{s}^{t_{j}} \mathbb{E}\left[\left|X_{r}^{-3} \phi^{2}\left(X_{r}\right)\right|^{2}\right] \mathrm{d} r \\
& +8 \lambda(\lambda h+1) \varepsilon_{0}^{-2}\left(\int_{s^{+}}^{t_{j}} \mathbb{E}\left[\left|\mathbb{1}_{\left\{X_{r^{-}}<1\right\}} \frac{\varphi\left(X_{r^{-}}\right)}{X_{r^{-}}^{2}}\right|^{2}\right] \mathrm{d} r+\int_{s^{+}}^{t_{j}} \mathbb{E}\left[\left|\mathbb{1}_{\left\{X_{r^{-}} \geq 1\right\}} \frac{\varphi\left(X_{r^{-}}\right)}{X_{r^{-}}}\right|^{2}\right] \mathrm{d} r\right) \\
& \leq 4 h \int_{s}^{t_{j}} \mathbb{E}\left[\left|\mu\left(X_{r}\right) X_{r}^{-2}\right|^{2}\right] \mathrm{d} r+4 \int_{s}^{t_{j}} \mathbb{E}\left[\left|\phi\left(X_{r}\right) X_{r}^{-2}\right|^{2}\right] \mathrm{d} r+4 h \int_{s}^{t_{j}} \mathbb{E}\left[\left|X_{r}^{-3} \phi^{2}\left(X_{r}\right)\right|^{2}\right] \mathrm{d} r \\
& +C \int_{s^{+}}^{t_{j}}\left(1+\mathbb{E}\left[\left|X_{r^{-}}\right|^{-2}\right]+\mathbb{E}\left[\left|X_{r^{-}}\right|^{-4}\right]\right) \mathrm{d} r \\
& \leq C h \text {. }
\end{aligned}
$$

Gathering the above estimates together implies that

$$
\left\|\int_{t_{j-1}}^{t_{j}} \mu\left(X_{t_{j}}\right)-\mu\left(X_{s}\right) \mathrm{d} s\right\|_{L^{2}(\Omega ; \mathbb{R})} \leq C h^{\frac{3}{2}} .
$$

In a similar way together with the Itô isometry, one can show that

$$
\left\|\int_{t_{j-1}}^{t_{j}} X_{t_{j-1}}^{\theta}-X_{s}^{\theta} \mathrm{d} W_{s}\right\|_{L^{2}(\Omega ; \mathbb{R})}^{2}=\int_{t_{j-1}}^{t_{j}}\left\|X_{t_{j-1}}^{\theta}-X_{s}^{\theta}\right\|_{L^{2}(\Omega ; \mathbb{R})}^{2} \mathrm{~d} s \leq C h^{2} .
$$

Employing the Itô isometry, the Hölder inequality and (2) yields

$$
\begin{aligned}
& \left\|\int_{t_{j-1}}^{t_{j}} \varphi\left(X_{t_{j-1}}\right)-\varphi\left(X_{s^{-}}\right) \mathrm{d} N_{s}\right\|_{L^{2}(\Omega ; \mathbb{R})}^{2} \\
= & \left\|\int_{t_{j-1}}^{t_{j}} \varphi\left(X_{t_{j-1}}\right)-\varphi\left(X_{s^{-}}\right) \mathrm{d}\left(\tilde{N}_{s}+\lambda s\right)\right\|_{L^{2}(\Omega ; \mathbb{R})}^{2} \\
\leq & 2\left\|\int_{t_{j-1}}^{t_{j}} \varphi\left(X_{t_{j-1}}\right)-\varphi\left(X_{s^{-}}\right) \mathrm{d} \tilde{N}_{s}\right\|_{L^{2}(\Omega ; \mathbb{R})}^{2}+2 \lambda^{2}\left\|\int_{t_{j-1}}^{t_{j}} \varphi\left(X_{t_{j-1}}\right)-\varphi\left(X_{s^{-}}\right) \mathrm{d} s\right\|_{L^{2}(\Omega ; \mathbb{R})}^{2} \\
\leq & \left(2 \lambda+2 \lambda^{2} h\right) \int_{t_{j-1}}^{t_{j}}\left\|\varphi\left(X_{t_{j-1}}\right)-\varphi\left(X_{s^{-}}\right)\right\|_{L^{2}(\Omega ; \mathbb{R})}^{2} \mathrm{~d} s \\
\leq & C M^{2} \int_{t_{j-1}}^{t_{j}}\left\|X_{t_{j-1}}-X_{s^{-}}\right\|_{L^{2}(\Omega ; \mathbb{R})}^{2} \mathrm{~d} s \\
\leq & C h^{2} .
\end{aligned}
$$

In view of 60, 61 and 62, one can arrive at

$$
\left\|\mathcal{M}_{t_{j}}\right\|_{L^{2}(\Omega ; \mathbb{R})} \leq C h .
$$

In order to bound $\left\|\mathbb{E}\left(\mathcal{M}_{t_{j}} \mid \mathcal{F}_{t_{j-1}}\right)\right\|_{L^{2}(\Omega ; \mathbb{R})}$, we first note that

$$
\mathbb{E}\left[\int_{t_{j-1}}^{t_{j}} b\left(X_{t_{j-1}}^{\theta}-X_{s}^{\theta}\right) \mathrm{d} W_{s} \mid \mathcal{F}_{t_{j-1}}\right]=0
$$


and use the compensated Poisson process and the Jensen type inequality to get

$$
\begin{aligned}
& \left\|\mathbb{E}\left(\int_{t_{j-1}}^{t_{j}} \varphi\left(X_{t_{j-1}}\right)-\varphi\left(X_{s^{-}}\right) \mathrm{d} N_{s} \mid \mathcal{F}_{t_{j-1}}\right)\right\|_{L^{2}(\Omega ; \mathbb{R})}^{2} \\
\leq & 2\left\|\mathbb{E}\left(\int_{t_{j-1}}^{t_{j}} \varphi\left(X_{t_{j-1}}\right)-\varphi\left(X_{s^{-}}\right) \mathrm{d} \tilde{N}_{s} \mid \mathcal{F}_{t_{j-1}}\right)\right\|_{L^{2}(\Omega ; \mathbb{R})}^{2} \\
& +2\left\|\lambda \mathbb{E}\left(\int_{t_{j-1}}^{t_{j}} \varphi\left(X_{t_{j-1}}\right)-\varphi\left(X_{s^{-}}\right) \mathrm{d} s \mid \mathcal{F}_{t_{j-1}}\right)\right\|_{L^{2}(\Omega ; \mathbb{R})}^{2} \\
\leq & 2 \lambda^{2} h \int_{t_{j-1}}^{t_{j}}\left\|\varphi\left(X_{t_{j-1}}\right)-\varphi\left(X_{s^{-}}\right)\right\|_{L^{2}(\Omega ; \mathbb{R})}^{2} \mathrm{~d} s \\
\leq & C M^{2} h \int_{t_{j-1}}^{t_{j}}\left\|X_{t_{j-1}}-X_{s^{-}}\right\|_{L^{2}(\Omega ; \mathbb{R})}^{2} \mathrm{~d} s \\
\leq & C h^{3} .
\end{aligned}
$$

Furthermore, the Jensen inequality together with 60 implies

$$
\left\|\mathbb{E}\left(\int_{t_{j-1}}^{t_{j}} \mu\left(X_{t_{j}}\right)-\mu\left(X_{s}\right) \mathrm{d} s \mid \mathcal{F}_{t_{j-1}}\right)\right\|_{L^{2}(\Omega ; \mathbb{R})} \leq\left\|\int_{t_{j-1}}^{t_{j}} \mu\left(X_{t_{j}}\right)-\mu\left(X_{s}\right) \mathrm{d} s\right\|_{L^{2}(\Omega ; \mathbb{R})} \leq C h^{\frac{3}{2}} .
$$

Armed with (64), 65 and (66), one can directly derive that

$$
\left\|\mathbb{E}\left(\mathcal{M}_{t_{j}} \mid \mathcal{F}_{t_{j-1}}\right)\right\|_{L^{2}(\Omega ; \mathbb{R})} \leq C h^{\frac{3}{2}} .
$$

Plugging (63) and (67) into (55) finishes the proof of Theorem 1 .

3.2 The critical case $\gamma+1=2 \theta$

Next we turn to the error analysis of the considered scheme for the model in the critical case $\gamma+1=2 \theta$. Similar to Lemma 4, we first get the next lemma.

Lemma 5 Let all conditions in Proposition 1 hold with model parameters satisfying $\gamma+1=2 \theta$ and $\frac{a_{2}}{b^{2}}>2 \gamma-\frac{3}{2}$. Let $\left\{X_{t}\right\}_{t \geq 0}$ be the process defined by (5). Then it holds that

$$
\left\|X_{t}-X_{s}\right\|_{L^{2}(\Omega ; \mathbb{R})} \leq C|t-s|^{\frac{1}{2}}
$$

Proof of Lemma 5. Similar to the proof of Lemma 4, one can show that

$$
\begin{aligned}
\mathbb{E}\left[\left|X_{t}-X_{s}\right|^{2}\right] \leq & 4|t-s| \int_{s}^{t} \mathbb{E}\left[\left|\mu\left(X_{r}\right)\right|^{2}\right] \mathrm{d} r+4 \int_{s}^{t} \mathbb{E}\left[\left|\phi\left(X_{r}\right)\right|^{2}\right] \mathrm{d} r+4 \int_{s}^{t} \mathbb{E}\left[\left|\varphi\left(X_{r^{-}}\right)\right|^{2}\right] \mathrm{d} r \\
& +4 \lambda^{2}|t-s| \int_{s}^{t} \mathbb{E}\left[\left|\varphi\left(X_{r^{-}}\right)\right|^{2}\right] \mathrm{d} r
\end{aligned}
$$

It is easy to check that

$$
\frac{2 a_{2}+b^{2}}{b^{2}}=\frac{2 a_{2}}{b^{2}}+1>4 \gamma-2>2 \gamma>2 \theta
$$

under the condition $\frac{a_{2}}{b^{2}}>2 \gamma-\frac{3}{2}$. With the aid of Lemmas 1,2 , we can further deduce that

$$
\begin{aligned}
\mathbb{E}\left[\left|X_{t}-X_{s}\right|^{2}\right] \leq & C|t-s| \int_{s}^{t}\left(1+\mathbb{E}\left[\left|X_{r}\right|^{-2}\right]+\mathbb{E}\left[\left|X_{r}\right|^{2}\right]+\mathbb{E}\left[\left|X_{r}\right|^{2 \gamma}\right]\right) \mathrm{d} r \\
& +C \int_{s}^{t} \mathbb{E}\left[\left|X_{r}\right|^{2 \theta}\right] \mathrm{d} r+C(1+|t-s|) \int_{s}^{t}\left(1+\mathbb{E}\left[\left|X_{r^{-}}\right|^{2}\right]\right) \mathrm{d} r \\
\leq & C|t-s|^{2}+C|t-s|+C|t-s|+C|t-s|^{2} \\
\leq & C|t-s|
\end{aligned}
$$

The proof is finished. 
Theorem 2 Let all conditions in Proposition 1 hold with model parameters satisfying $\gamma+1=2 \theta$ and $\frac{a_{2}}{b^{2}}>2 \gamma-\frac{3}{2}$. Let $\left\{X_{t}\right\}_{0 \leq t \leq T}$ and $\left\{Y_{n}\right\}_{0 \leq n \leq N}$ be solutions to (1) and (27), respectively. Then for any $h<\frac{1}{2 a_{1}}$ we have

$$
\sup _{N \in \mathbb{N}} \sup _{0 \leq n \leq N}\left\|Y_{n}-X_{t_{n}}\right\|_{L^{2}(\Omega ; \mathbb{R})} \leq C h^{\frac{1}{2}}
$$

Proof of Theorem 2. The proof here follows the same lines in the proof of Theorem 1 for the case $\gamma+1>2 \theta$. Clearly, equalities (34)-(38) still hold for the critical case $\gamma+1=2 \theta$ and we need to estimate (39) first. Similar to (40), we have

$$
\begin{aligned}
\mathbb{E}\left[\left|\mathcal{M}_{t_{n}}\right|^{2}\right] \leq & 4 h \int_{t_{n-1}}^{t_{n}} \mathbb{E}\left[\left|\mu\left(X_{t_{n}}\right)-\mu\left(X_{s}\right)\right|^{2}\right] \mathrm{d} s+4 \int_{t_{n-1}}^{t_{n}} \mathbb{E}\left[\left|\phi\left(X_{t_{n-1}}\right)-\phi\left(X_{s}\right)\right|^{2}\right] \mathrm{d} s \\
& +4 \lambda \int_{t_{n-1}}^{t_{n}} \mathbb{E}\left[\left|\varphi\left(X_{t_{n-1}}\right)-\varphi\left(X_{s^{-}}\right)\right|^{2}\right] \mathrm{d} s+4 \lambda^{2} h \int_{t_{n-1}}^{t_{n}} \mathbb{E}\left[\left|\varphi\left(X_{t_{n-1}}\right)-\varphi\left(X_{s^{-}}\right)\right|^{2}\right] \mathrm{d} s \\
\leq & 24 h \int_{t_{n-1}}^{t_{n}} a_{-1}^{2}\left(\mathbb{E}\left[\left|X_{t_{n}}^{-1}\right|^{2}\right]+\mathbb{E}\left[\left|X_{s}^{-1}\right|^{2}\right]\right)+a_{1}^{2}\left(\mathbb{E}\left[\left|X_{t_{n}}\right|^{2}\right]+\mathbb{E}\left[\left|X_{s}\right|^{2}\right]\right) \\
& +a_{2}^{2}\left(\mathbb{E}\left[\left|X_{t_{n}}\right|^{2 \gamma}\right]+\mathbb{E}\left[\left|X_{s}\right|^{2 \gamma}\right]\right) \mathrm{d} s+8 b^{2} \int_{t_{n-1}}^{t_{n}} \mathbb{E}\left[\left|X_{t_{n-1}}\right|^{2 \theta}\right]+\mathbb{E}\left[\left|X_{s}\right|^{2 \theta}\right] \mathrm{d} s \\
& +8 \lambda M^{2}(1+\lambda h) \int_{t_{n-1}}^{t_{n}} \mathbb{E}\left[\left|X_{t_{n-1}}\right|^{2}\right]+\mathbb{E}\left[\left|X_{s^{-}}\right|^{2}\right] \mathrm{d} s .
\end{aligned}
$$

This together with 70 and Lemmas 1, 2 implies that

$$
\mathbb{E}\left[\left|\mathcal{M}_{t_{n}}\right|^{2}\right]<\infty
$$

Since $\frac{a_{2}}{b^{2}}>2 \gamma-\frac{3}{2}$, we can find some $q>2$ such that

$$
\begin{aligned}
\mu^{\prime}(x)+\frac{q-1}{2}\left|\phi^{\prime}(x)\right|^{2} & =-a_{-1} x^{-2}+a_{1}-a_{2} \gamma x^{\gamma-1}+\frac{q-1}{2} b^{2} \theta^{2} x^{2 \theta-2} \\
& <a_{1}+\left(\frac{q-1}{2} b^{2} \theta^{2}-a_{2} \gamma\right) x^{\gamma-1} \\
& \leq a_{1}, \quad x \in \mathbb{R}_{+}
\end{aligned}
$$

which implies

$$
\langle x-y, \mu(x)-\mu(y)\rangle+\frac{q-1}{2}|\phi(x)-\phi(y)|^{2} \leq a_{1}|x-y|^{2}, \quad \forall x, y \in \mathbb{R}_{+} .
$$

With this, one can also rely on the mathematical induction argument to acquire (44) and (52) for the critical case. Then repeating the same arguments used in (53)-(54) one can arrive at (55) for the critical case. So it remains to estimate $\mathbb{E}\left[\left|\mathcal{M}_{t_{j}}\right|^{2}\right]$ and $\mathbb{E}\left[\left|\mathbb{E}\left(\mathcal{M}_{t_{j}} \mid \mathcal{F}_{t_{j-1}}\right)\right|^{2}\right], j=1,2, \cdots, n$. According to $\left[56\right.$, the estimate of $\mathbb{E}\left[\left|\mathcal{M}_{t_{j}}\right|^{2}\right]$ relies on treating $\left\|X_{t_{j}}^{\gamma}-X_{s}^{\gamma}\right\|_{L^{2}(\Omega ; \mathbb{R})}^{2},\left\|X_{t_{j}}^{-1}-X_{s}^{-1}\right\|_{L^{2}(\Omega ; \mathbb{R})}^{2}$ and 
$\left\|\int_{t_{j-1}}^{t_{j}} X_{t_{j-1}}^{\theta}-X_{s}^{\theta} \mathrm{d} W_{s}\right\|_{L^{2}(\Omega ; \mathbb{R})}^{2}$ separately. Owing to (3), (58), (70) and Lemmas 1 , we have

$$
\begin{aligned}
& \left\|X_{t_{j}}^{\gamma}-X_{s}^{\gamma}\right\|_{L^{2}(\Omega ; \mathbb{R})}^{2} \\
\leq & 4 \gamma^{2} h \int_{s}^{t_{j}} \mathbb{E}\left[\left|\mu\left(X_{r}\right) X_{r}^{\gamma-1}\right|^{2}\right] \mathrm{d} r+4 \gamma^{2} \int_{s}^{t_{j}} \mathbb{E}\left[\left|\phi\left(X_{r}\right) X_{r}^{\gamma-1}\right|^{2}\right] \mathrm{d} r \\
& +\gamma^{2}(\gamma-1)^{2} h \int_{s}^{t_{j}} \mathbb{E}\left[\left|X_{r}^{\gamma-2} \phi^{2}\left(X_{r}\right)\right|^{2}\right] \mathrm{d} r+8 \lambda(\lambda h+1) \int_{s^{+}}^{t_{j}} \mathbb{E}\left[\left|\left(X_{r^{-}}+\varphi\left(X_{r^{-}}\right)\right)^{\gamma}-X_{r^{-}}^{\gamma}\right|^{2}\right] \mathrm{d} r \\
\leq & C h \int_{s}^{t_{j}}\left(\mathbb{E}\left[\left|X_{r}\right|^{2 \gamma-4}\right]+\mathbb{E}\left[\left|X_{r}\right|^{2 \gamma-2}\right]+\mathbb{E}\left[\left|X_{r}\right|^{2 \gamma}\right]+\mathbb{E}\left[\left|X_{r}\right|^{4 \gamma-2}\right]\right) \mathrm{d} r \\
& +4 b^{2} \gamma^{2} \int_{s}^{t_{j}} \mathbb{E}\left[\left|X_{r}\right|^{3 \gamma-1}\right] \mathrm{d} r+\gamma^{2}(\gamma-1)^{2} b^{2} h \int_{s}^{t_{j}} \mathbb{E}\left[\left|X_{r}\right|^{4 \gamma-2}\right] \mathrm{d} r \\
& +C \int_{s^{+}}^{t_{j}}\left(\mathbb{E}\left[\left|X_{r^{-}}\right|^{2 \gamma}\right]+\mathbb{E}\left[\left|\varphi\left(X_{r^{-}}\right)\right|^{2 \gamma}\right]\right) \mathrm{d} r \\
\leq & C h \int_{s}^{t_{j}}\left(\mathbb{E}\left[\left|X_{r}\right|^{2 \gamma-4}\right]+\mathbb{E}\left[\left|X_{r}\right|^{2 \gamma-2}\right]+\mathbb{E}\left[\left|X_{r}\right|^{2 \gamma}\right]+\mathbb{E}\left[\left|X_{r}\right|^{4 \gamma-2}\right]\right) \mathrm{d} r \\
& +4 b^{2} \gamma^{2} \int_{s}^{t_{j}} \mathbb{E}\left[\left|X_{r}\right|^{3 \gamma-1}\right] \mathrm{d} r+\gamma^{2}(\gamma-1)^{2} b^{2} h \int_{s}^{t_{j}} \mathbb{E}\left[\left|X_{r}\right|^{4 \gamma-2}\right] \mathrm{d} r+C \int_{s^{+}}^{t_{j}}\left(1+\mathbb{E}\left[\left|X_{r^{-}}\right|^{2 \gamma}\right]\right) \mathrm{d} r \\
\leq & C h .
\end{aligned}
$$

In the same manner as above, we derive from $(59)$ that

$$
\begin{aligned}
& \left\|X_{t_{j}}^{-1}-X_{s}^{-1}\right\|_{L^{2}(\Omega ; \mathbb{R})}^{2} \\
\leq & 4 h \int_{s}^{t_{j}} \mathbb{E}\left[\left|\mu\left(X_{r}\right) X_{r}^{-2}\right|^{2}\right] \mathrm{d} r+4 \int_{s}^{t_{j}} \mathbb{E}\left[\left|\phi\left(X_{r}\right) X_{r}^{-2}\right|^{2}\right] \mathrm{d} r \\
& +4 h \int_{s}^{t_{j}} \mathbb{E}\left[\left|X_{r}^{-3} \phi^{2}\left(X_{r}\right)\right|^{2}\right] \mathrm{d} r+8 \lambda \int_{s^{+}}^{t_{j}} \mathbb{E}\left[\left|\frac{\varphi\left(X_{r^{-}}\right)}{\left(X_{r^{-}}+\varphi\left(X_{r^{-}}\right) X_{r^{-}}\right.}\right|^{2}\right] \mathrm{d} r \\
& +8 \lambda^{2} h \int_{s^{+}}^{t_{j}} \mathbb{E}\left[\left|\frac{\varphi\left(X_{r^{-}}\right)}{\left(X_{r^{-}}+\varphi\left(X_{r^{-}}\right)\right) X_{r^{-}}}\right|^{2}\right] \mathrm{d} r \\
\leq & C h \int_{s}^{t_{j}} \mathbb{E}\left[\left|X_{r}\right|^{-6}\right]+\mathbb{E}\left[\left|X_{r}\right|^{-4}\right]+\mathbb{E}\left[\left|X_{r}\right|^{-2}\right]+\mathbb{E}\left[\left|X_{r}\right|^{2 \gamma-4}\right] \mathrm{d} r \\
& +C \int_{s}^{t_{j}} \mathbb{E}\left[\left|X_{r}\right|^{2 \theta-4}\right] \mathrm{d} r+C \int_{s}^{t_{j}} \mathbb{E}\left[\left|X_{r}\right|^{4 \theta-6}\right] \mathrm{d} r \\
& +8 \lambda(\lambda h+1) \varepsilon_{0}^{-2}\left(\int_{s^{+}}^{t_{j}} \mathbb{E}\left[\left|\mathbb{1}_{\left\{X_{r^{-}}<1\right\}} \frac{\varphi\left(X_{r^{-}}\right)}{X_{r^{-}}^{2}}\right|^{2}\right] \mathrm{d} r+\int_{s^{+}}^{t_{j}} \mathbb{E}\left[\left|\mathbb{1}_{\left\{X_{r^{-}} \geq 1\right\}} \frac{\varphi\left(X_{r^{-}}\right)}{X_{r^{-}}}\right|^{2}\right] \mathrm{d} r\right) \\
\leq & C h \int_{s}^{t_{j}} \mathbb{E}\left[\left|X_{r}\right|^{-6}\right]+\mathbb{E}\left[\left|X_{r}\right|^{-4}\right]+\mathbb{E}\left[\left|X_{r}\right|^{-2}\right]+\mathbb{E}\left[\left|X_{r}\right|^{2 \gamma-4}\right] \mathrm{d} r \\
& +C \int_{s}^{t_{j}} \mathbb{E}\left[\left|X_{r}\right|^{2 \theta-4}\right] \mathrm{d} r+C \int_{s}^{t_{j}} \mathbb{E}\left[\left|X_{r}\right|^{4 \theta-6}\right] \mathrm{d} r+C \int_{s^{+}}^{t_{j}}\left(1+\mathbb{E}\left[\left|X_{r^{-}}\right|^{-2}+\left|X_{r^{-}}\right|^{-4}\right]\right) \mathrm{d} r \\
\leq & C h .
\end{aligned}
$$

Similar to (77), we have

$$
\left\|X_{t_{j}}^{\theta}-X_{s}^{\theta}\right\|_{L^{2}(\Omega ; \mathbb{R})}^{2} \leq C h .
$$

Therefore, for $\gamma+1=2 \theta$ and $\frac{a_{2}}{b^{2}}>2 \gamma-\frac{3}{2}$, it holds that

$$
\left\|\mathcal{M}_{t_{j}}\right\|_{L^{2}(\Omega ; \mathbb{R})} \leq C h .
$$

Following similar arguments as used in 67 and considering $\gamma+1=2 \theta, \frac{a_{2}}{b^{2}}>2 \gamma-\frac{3}{2}$, we can obtain

$$
\left\|\mathbb{E}\left(\mathcal{M}_{t_{j}} \mid \mathcal{F}_{t_{j-1}}\right)\right\|_{L^{2}(\Omega ; \mathbb{R})} \leq C h^{\frac{3}{2}}
$$


Based on (55), we can easily derive that

$$
\sup _{N \in \mathbb{N}} \sup _{0 \leq n \leq N}\left\|Y_{n}-X_{t_{n}}\right\|_{L^{2}(\Omega ; \mathbb{R})} \leq C h^{\frac{1}{2}}
$$

as required.

\section{Numerical results}

In the present section, we provide some numerical experiments to support the previous findings. We consider a generalized Ait-sahalia-type rate model with Poisson jumps of the form

$$
\mathrm{d} X_{t}=\left(a_{-1} X_{t}^{-1}-a_{0}+a_{1} X_{t}-a_{2} X_{t}^{\gamma}\right) \mathrm{d} t+b X_{t}^{\theta} \mathrm{d} W_{t}+\varphi\left(X_{t^{-}}\right) \mathrm{d} N_{t}, \quad X_{0}=1>0,
$$

with $a_{-1}, a_{0}, a_{1}, a_{2}, b>0$ and $\gamma, \theta>1$. In the following tests we take two sets of model parameters:

- Case 1: $a_{-1}=2, a_{0}=1, a_{1}=1.5, a_{2}=5, b=1, \theta=2, \gamma=3.5, \lambda=1$;

- Case 2: $a_{-1}=2, a_{0}=1, a_{1}=1.5, a_{2}=5, b=1, \theta=2, \gamma=3, \lambda=1$.

The first set of parameters satisfies $\gamma+1>2 \theta$ and the second one belongs to the critical case $\gamma+1=2 \theta$. As the first part of numerical results, we list in Table 1 the percentage of negative paths by using the explicit Euler method (EM) and the BEM with three time stepsizes $h=\frac{1}{4}, \frac{1}{8}, \frac{1}{16}$ over $10^{5}$ paths. It is clear that the EM method fails to preserve the positivity, although the percentage of negative paths decreases as the stepsize becomes smaller. However, as expected, the BEM remains positive for all stepsize, which is consistent with the previous theoretical results.

\begin{tabular}{cccccc}
\hline Stepsize $(T=1)$ & $\varphi(x)$ & EM (Case 1) & EM (Case 2) & BEM (Case 1) & BEM (Case 2) \\
\hline \multirow{2}{*}{$\frac{1}{4}$} & $-0.2 x$ & $89.91 \%$ & $86.75 \%$ & 0 & 0 \\
& $x$ & $91.48 \%$ & $88.46 \%$ & 0 & 0 \\
\multirow{2}{*}{$\frac{1}{8}$} & $\sin (x)$ & $91,09 \%$ & $88.03 \%$ & 0 & 0 \\
\hline \multirow{2}{*}{$\frac{1}{16}$} & $-0.2 x$ & $47.81 \%$ & $40.52 \%$ & 0 & 0 \\
& $\sin (x)$ & $71.51 \%$ & $64.31 \%$ & 0 & 0 \\
\hline
\end{tabular}

Table 1: The percentage of negative paths for EM and BEM with three stepsizes over $10^{5}$ paths.

In the next step, we are to test the mean-square convergence rate of BEM for six different stepsizes $h=2^{-j}, j=7,8, \cdots, 11$. As usual, the expectation is approximated by using 10000 Brownian and Poisson paths and the "true" solution of the model is identified with the numerical one using a small stepsize $h_{\text {exact }}=2^{-13}$. In order to clearly display the convergence rates, for various choices of the jump coefficient function $\varphi$ we depict in Figures 1, 2, 3 mean-square approximation errors against six different stepsizes on a log-log scale. From Figures 1, 2, 3, one can observe that the mean-square error (solid lines) and the reference (dashed lines) match well, which indicates the mean-square convergence rate of order one-half.

\section{References}

1. A. Andersson AND R. KRUSE, Mean-square convergence of the BDF2-Maruyama and backward Euler schemes for SDE satisfying a global monotonicity condition, BIT Numer. Math., 57 (2017), pp. 21-53.

2. E. BuckWAR AND M. RiEDleR, Runge-kutta methods for jump-diffusion differential equations, J. Comput. Appl. Math., 236 (2011), pp. 1155-1182. 

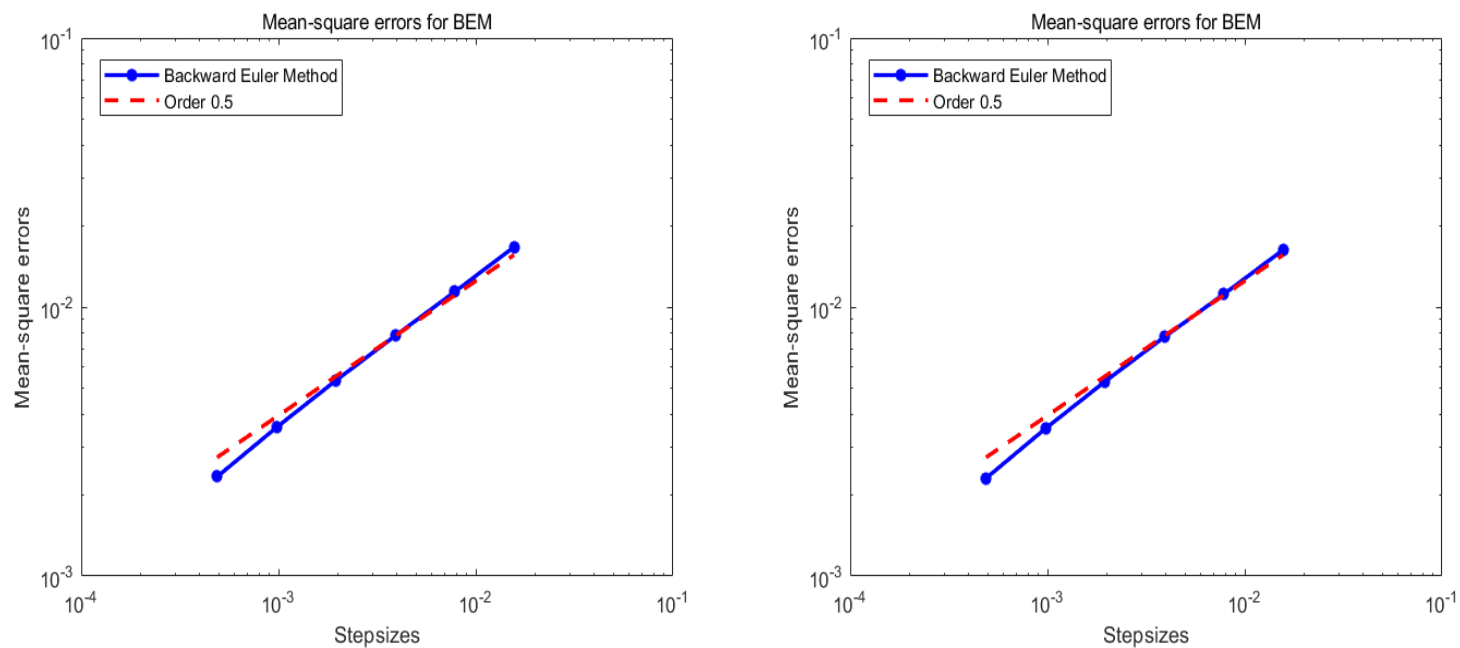

Fig. 1: Numerical results for (83) with $T=1$ : Mean-square convergence rate of BEM with $\varphi(x)=-0.2 x$ for Case 1 (Left) and Case 2 (Right).
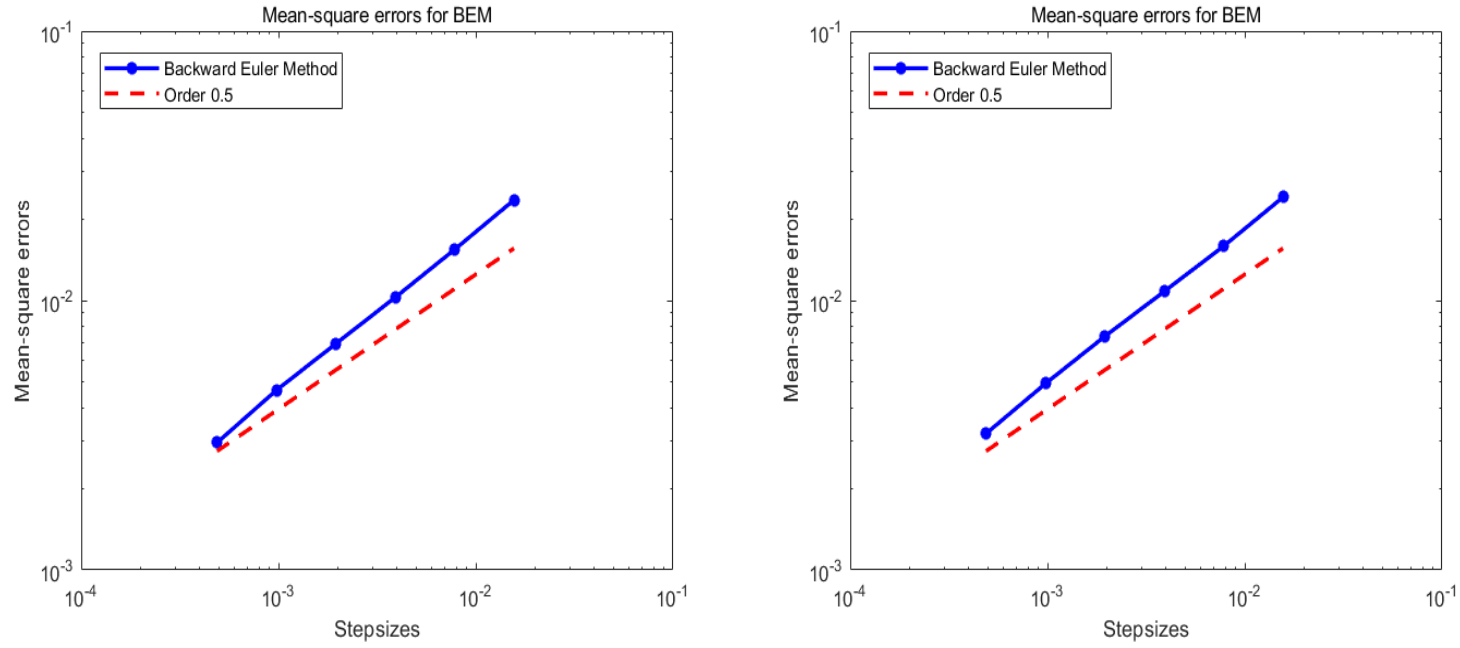

Fig. 2: Numerical results for (83) with $T=1$ : Mean-square convergence rate of BEM with $\varphi(x)=x$ for Case 1 (Left) and Case 2 (Right).

3. G. Chalmers And D. Higham, Asymptotic stability of a jump-diffusion equation and its numerical approximation, SIAM J. Sci. Comput., 31 (2008), pp. 1141-1155.

4. Z. Chen AND S. GAN, Convergence and stability of the backward Euler method for jump-diffusion SDEs with super-linearly growing diffusion and jump coefficients, J. Comput. Appl. Math., 365 (2020), pp. 350-369.

5. Z. Chen, S. GAn, AND X. WANG, Mean-square approximations of Lévy noise driven SDEs with super-linearly growing diffusion and jump coefficients, Discrete Contin. Dynam. Systems-B., 24 (2019), pp. 4513-4545.

6. R. Cont And P. TAnkov, Financial Modelling with Jump Processes, Chapman and Hall/CRC, 2004.

7. K. Dareiotis, C. Kumar, And S. Sabanis, On tamed Euler approximations of SDEs driven by Lévy noise with applications to delay equations, SIAM J. Numer. Anal., 54 (2016), pp. 1840-1872.

8. S. Deng, C. Fei, W. Fei, And X. MaO, Generalized Ait-Sahalia-type interest rate model with 

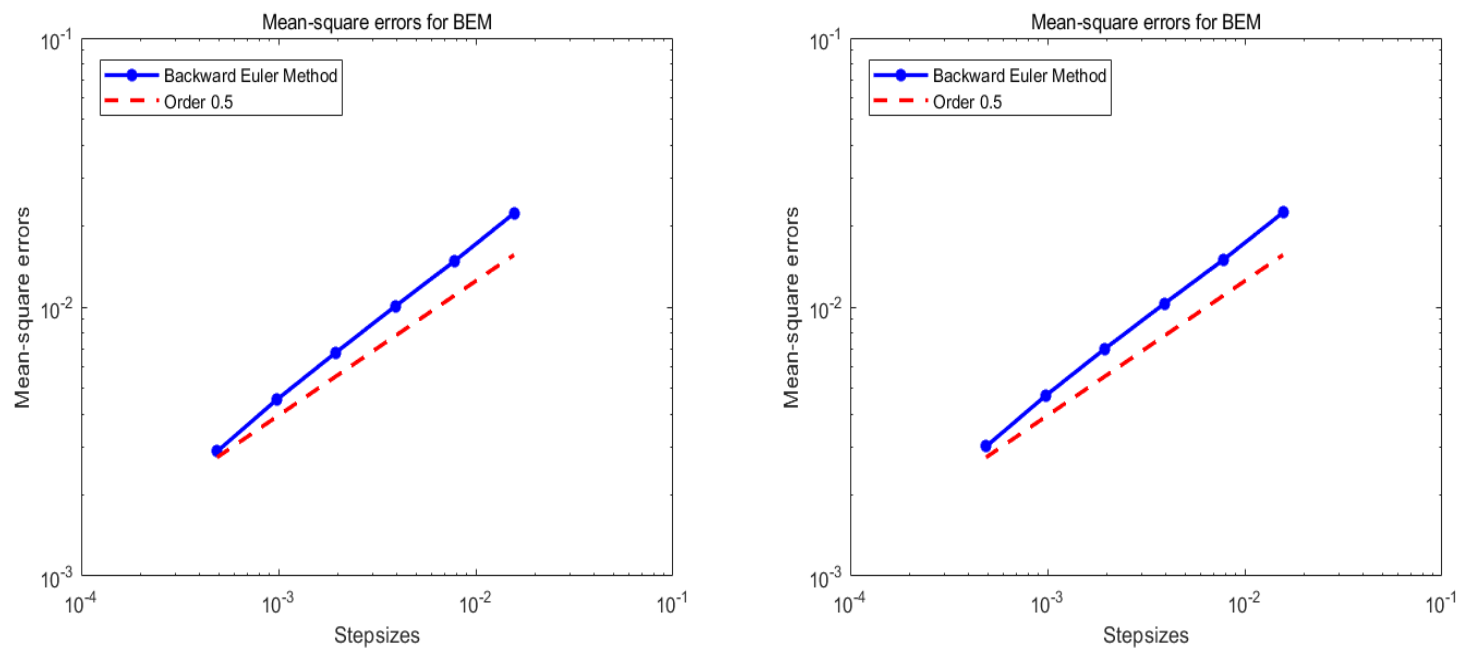

Fig. 3: Numerical results for 83 with $T=1$ : Mean-square convergence rate of BEM with $\varphi(x)=\sin (x)$ for Case 1 (Left) and Case 2 (Right).

Poisson jumps and convergence of the numerical approximation, Physica A., 533 (2019), p. 122057.

9. S. Deng, W. FEI, W. LiU, And X. MaO, The truncated EM method for stochastic differential equations with Poisson jumps, J. Comput. Appl. Math., 355 (2019), pp. 232-257.

10. A. GARDOŃ, The order of approximations for solutions of Itô-type stochastic differential equations with jumps, Stoch Anal. Appl., 22 (2004), pp. 679-699.

11. D. Higham AND P. KloEden, Numerical methods for nonlinear stochastic differential equations with jumps, Numer. Math., 101 (2005), pp. 101-119.

12. D. Higham And P. Kloeden, Convergence and stability of implicit methods for jump diffusion systems, Int. J. Numer. Anal. Model, 3 (2006), pp. 125-140.

13. D. Higham And P. Kloeden, Strong convergence rates for backward Euler on a class of nonlinear jump-diffusion problems, J. Comput. Appl. Math., 205 (2007), pp. 949-956.

14. D. Higham, X. Mao, And A. Stuart, Strong convergence of Euler-type methods for nonlinear stochastic differential equations, SIAM J. Numer. Anal., 40 (2002), pp. 1041-1063.

15. C. HuAng, Exponential mean square stability of numerical methods for systems of stochastic differential equations, J. Comput. Appl. Math., 236 (2012), pp. 4016-4026.

16. A. Kaluża And P. PRZybylowicz, Optimal global approximation of jump-diffusion SDEs via pathindependent step-size control, Appl. Numer. Math., 128 (2018), pp. 24-42.

17. C. Kumar And S. SABAnis, On explicit approximations for Lévy driven SDEs with super-linear diffusion coefficients, Electron. J. Probab., 22 (2017), pp. 1-19.

18. C. Kumar And S. Sabanis, On tamed Milstein schemes of SDEs driven by Lévy noise, Discrete Contin. Dynam. Systems-B., 22 (2017), pp. 421-463.

19. L. LI AND T. DAI, On a positivity preserving numerical scheme for jump-extended CIR process: the Alpha-Stable Case, BIT Numer. Math., 59 (2019), pp. 747-774.

20. X. Mao, Stochastic Differential Equations and Applications, Horwood, 2008.

21. X. MAO AND L. SzPruch, Strong convergence and stability of implicit numerical methods for stochastic differential equations with non-globally lipschitz continuous coefficients, J. Comput. Appl. Math., 238 (2013), pp. 14-28.

22. X. Mao AND L. SzPruch, Strong convergence rates for backward EulerMaruyama method for nonlinear dissipative-type stochastic differential equations with super-linear diffusion coefficients, Stoch., 85 (2013), pp. 144-171.

23. A. NeUEnKIRCh AND L. SzPruch, First order strong approximations of scalar SDEs defined in a domain, Numer. Math., 128 (2014), pp. 103-136.

24. E. Platen AND N. Bruti-LiBerati, Numerical solution of stochastic differential equations with jumps in finance, Springer Science \& Business Media, 2010. 
25. P. Przybylowicz, Optimal global approximation of stochastic differential equations with additive Poisson noise, Numer. Algor., 73 (2016), pp. 323-348.

26. L. Szpruch, X. Mao, D. Higham, and J. Pan, Numerical simulation of a strongly nonlinear Ait-Sahalia-type interest rate model, BIT Numer. Math., 51 (2011), pp. 405-425.

27. X. WANG AND S. GAN, Compensated stochastic theta methods for stochastic differential equations with jumps, Appl. Numer. Math., 60 (2010), pp. 877-887.

28. X. WAng, J. Wu, AND B. Dong, Mean-square convergence rates of stochastic theta methods for SDEs under a coupled monotonicity condition, BIT Numer. Math., 60 (2020), pp. 759-790.

29. X. YANG AND X. WANG, A transformed jump-adapted backward Euler method for jump-extended CIR and CEV models, Numer. Algor., 71 (2017), pp. 39-57.

30. X. ZONG AND F. WU, Choice of $\theta$ and mean-square exponential stability in the stochastic theta method of stochastic differential equations, J. Comput. Appl. Math., 255 (2014), pp. 837-847. 